\title{
HALF DENSITY VOLUMES OF REPRESENTATION SPACES OF SOME 3-MANIFOLDS AND THEIR APPLICATION
}

\author{
JINSUNG PARK \\ Department of mathematics \\ Seoul National University \\ 151-742 Seoul Korea
}

\begin{abstract}
In this paper we compute half density volumes of the irreducible $S U(2)$ representation spaces for Seifert fibred manifolds and graph manifolds. These representation spaces are not discrete so that the half density derived from the Reidemeister torsion is used as a measure. As an application of our result we get the exact value of the Jeffrey-Weitsman-Witten invariant of a Seifert fibred manifold with non-discrete irreducible $S U(2)$-representation space.
\end{abstract}

\section{$\S 1$ Introduction}

In this paper we compute half density volumes of the irreducible $S U(2)$-representation spaces of Seifert fibred manifolds and graph manifolds. The half density over the irreducible $S U(2)$-representation space of a 3-manifold comes from the Reidemeister torsion for Ad-SU(2)-representation. More precisely the determinant term of the first homology of the Reidemeister torsion gives the half density of the irreducible representation space. This is because the tangent space of the irreducible representation space can be identified with the first cohomology of the twisted cochain complex.

The motivation of this paper is given by two sources. The first is the E.Witten's method to compute the symplectic volume of the irreducible $S U(2)$-representation space of a Riemann surface. In [W2] Witten suggested an useful method to compute the symplectic volume of this space using the Reidemeister torsion and the character theory of the Lie group $S U(2)$. The second is the invariant defined by L.C.Jeffrey and J.Weitsman in $[\mathrm{J}, \mathrm{W} 1]$. This invariant is motivated by the 
asymptotic expansion of the Witten invariant of a 3-manifold. They define this invariant using the Reidemeister torsion as a half density measure of the irreducible $S U(2)$-representation space. Hence to compute this invariant we must compute the Reidemeister torsion completely including the determinant term of the homology which is the half density measure of the $S U(2)$-representation space. In this paper we call this invariant as the Jeffrey-Weitsman-Witten invariant. From the above motivations we could consider naturally that this invariant might be computed by the method of [W2]. The examples to which we apply the method of Witten are Seifert fibred manifolds and graph manifolds. This is because these manifolds are made from the trivial circle bundle over the Riemann surfaces by twisting finite fibers. So the method of Witten is applicable with some modification.

To compute the half density derived from the Reidemeister torsion, we must compute both the scalar part and the determinant part of the Reidemeister torsion. The method to compute the scalar part comes from $[\mathrm{F}]$. For Seifert fibred manifolds and graph manifolds this value gives the weight of the half density to each connected component of the irreducible $S U(2)$-representation space. So we combine two methods of $[\mathrm{F}]$ and [W2] to compute the half density volumes of the irreducible $S U(2)$-representation spaces for Seifert fibred manifolds and graph manifolds.

Our computing method is 'to cut and to paste' with a topological view point. This can be comparable to the method of P.Kirk and E.Klassen to compute the Chern-Simons invariants for 3-manifolds $[\mathrm{K}, \mathrm{K}]$. We decompose the given manifolds into simple pieces which we can deal easily and then we glue the data of the decomposed pieces and investigate the gluing maps. The data of the pieces and the gluing maps gives the result that we want to get.

Now we explain how this paper is organized. In the section 2 we study basic examples which are the building blocks of Seifert fibred manifolds and graph manifolds. We study the Reidemeister torsions and the $S U(2)$-representation spaces of these basic examples. In the section 3 we compute the scalar part of the Reidemeister torsions of Seifert fibred manifolds and graph manifolds for Ad-SU(2)representations. The computing method of the section 3 comes from $[\mathrm{F}]$. This method is exactly 'to cut and to paste' so that we can apply the result of the section 2 . In the section 4 we integrate the determinant term of the first homology part of the Reidemeister torsion over the irreducible $S U(2)$-representation space. The integration process of this section is also 'to cut and to paste'. We get the half density volume of the irreducible $S U(2)$-representation space by investigation of 
the pasting process for the half densities of the representation spaces of the decomposed pieces. In the section 5 we apply the result that we get in the section 4 to compute the Jeffrey-Weitsman-Witten invariant for Seifert fibred manifold whose irreducible $S U(2)$-representation space is non-discrete. This gives the exact value of the Jeffrey-Weitsman-Witten invariant for a Seifert fibred manifold with the non-discrete $S U(2)$-representation space combined with the result of D.R.Auckly for the Chern-Simons invariant for this manifold.

\section{$\S 2$ Basic Examples}

In this section we study some basic examples which we will use in section 3 and 4. We study the Reidemeister torsions and $S U(2)$-representation spaces of $S^{1}, T^{2}$ and pants $P$. These examples are the building blocks of some 3 -manifolds which we will deal later. From now on R-torsion means the Reidemeister torsion always. For the definition and the basic property of the R-torsion, see $[\mathrm{F}]$.

Our first example is the circle $S^{1}$. Every $S U(2)$-representation of $\pi_{1}\left(S^{1}\right)$ is reducible since $\pi_{1}\left(S^{1}\right)$ is abelian. Hence the representation is determined by the holonomy parameter $u$ so that the $S U(2)$-representation $\rho_{u}$ has the following form up to conjugation

$$
\rho_{u}(1)=\left(\begin{array}{cc}
e^{2 \pi i u} & 0 \\
0 & e^{-2 \pi i u}
\end{array}\right)
$$

for the generator $1 \in \pi_{1}\left(S^{1}\right)=Z$. Hence the $S U(2)$-representation space for $\pi_{1}\left(S^{1}\right)$ is $S^{1}$ which can be identified with the maximal torus $T^{1}$ of $S U(2)$. We denote this space by $\mathcal{L}$. This representation space will be used importantly later. The Rtorsion of $S^{1}$ for Ad- $S U(2)$-representation is denoted by $\tau\left(S^{1}, A d\left(\rho_{u}\right)\right)$. R-torsion is given by the torsion of the following chain complex $C .\left(S^{1}, A d\left(\rho_{u}\right)\right)$ :

$$
0 \rightarrow C_{1}\left(R^{1}\right) \otimes_{\pi} s u(2) \rightarrow C_{0}\left(R^{1}\right) \otimes_{\pi} s u(2) \rightarrow 0
$$

where $R^{1}$ is the universal covering space of $S^{1}$ and the tensor product is taken over the $\pi=\pi_{1}\left(S^{1}\right)$. Then the homology of $C .\left(S^{1}, A d\left(\rho_{u}\right)\right)$ is

$$
\begin{aligned}
& H_{0}\left(S^{1}, s u(2)_{\rho}\right)=R[e \otimes v] \\
& H_{1}\left(S^{1}, s u(2)_{\rho}\right)=R[x \otimes v]
\end{aligned}
$$


where $R$ is the real number field, $e, x$ are the 0,1 -cells of $S^{1}$ respectively and $v$ is the $\operatorname{Ad}(\rho)$-invariant vector in $s u(2)$. Then we have that

$$
\tau\left(S^{1}, A d\left(\rho_{u}\right)\right)=4 \sin ^{2}(2 \pi u) D_{H_{*}\left(S^{1}\right)}^{-1} \in \operatorname{det} H_{*}\left(S^{1}, s u(2)_{\rho}\right)^{-1}
$$

where $D_{H_{*}\left(S^{1}\right)}=(e \otimes v) \otimes(x \otimes v)^{-1}$ and $\operatorname{det} H_{*}\left(S^{1}, s u(2)_{\rho}\right)=\operatorname{det} H_{0}\left(S^{1}, s u(2)_{\rho}\right) \otimes$ $\operatorname{det} H_{1}\left(S^{1}, s u(2)_{\rho}\right)^{-1}$. For the notation about the determinant line, we follow the notation of $[\mathrm{F}]$.

The next example is the torus $T^{2}$. Since $\pi_{1}\left(T^{2}\right)$ is also abelian, every $S U(2)$ representation of $\pi_{1}\left(T^{2}\right)$ is reducible with a following form

$$
\rho_{\alpha, \beta}((1,0))=\left(\begin{array}{cc}
e^{2 \pi i \alpha} & 0 \\
0 & e^{-2 \pi i \alpha}
\end{array}\right), \quad \rho_{\alpha, \beta}((0,1))=\left(\begin{array}{cc}
e^{2 \pi i \beta} & 0 \\
0 & e^{-2 \pi i \beta}
\end{array}\right)
$$

for a basis $(1,0),(0,1)$ of $\pi_{1}\left(T^{2}\right)$. We know that $S U(2)$-representation space $R\left(T^{2}, S U(2)\right)$ is an object which is called as the 'pillow case'. The R-torsion $\tau\left(T^{2}, A d\left(\rho_{\alpha, \beta}\right)\right)$ is the torsion of the following chain complex $C .\left(T^{2}, A d\left(\rho_{\alpha, \beta}\right)\right)$ :

$$
0 \rightarrow C_{2}\left(R^{2}\right) \otimes_{\pi} s u(2) \rightarrow C_{1}\left(R^{2}\right) \otimes_{\pi} s u(2) \rightarrow C_{0}\left(R^{2}\right) \otimes_{\pi} s u(2) \rightarrow 0
$$

Then we have

$$
\begin{aligned}
& H_{0}\left(T^{2}, s u(2)_{\rho}\right)=R[e \otimes v], \\
& H_{1}\left(T^{2}, s u(2)_{\rho}\right)=R[x \otimes v, y \otimes v], \\
& H_{2}\left(T^{2}, s u(2)_{\rho}\right)=R[x \cup y \otimes v]
\end{aligned}
$$

where $R$ is the real number field, $v$ is the $A d(\rho)$-invariant vector in $s u(2)$ and $e, x, y, x \cup y$ are the $0,1,2$ cells of $T^{2}$ respectively. From the Poincare duality,

$$
\begin{aligned}
\tau\left(T^{2}, A d(\rho)\right) & =(e \otimes v)^{-1} \otimes(x \otimes v \wedge y \otimes v) \otimes(x \cup y \otimes v)^{-1} \\
& =D_{H_{*}\left(T^{2}\right)}^{-1} \in \operatorname{det} H_{*}\left(T^{2}, s u(2)_{\rho}\right)^{-1}
\end{aligned}
$$

where $\operatorname{det} H_{*}\left(T^{2}, s u(2)_{\rho}\right)=\otimes_{i=0}^{2} H_{i}\left(T^{2}, s u(2)_{\rho}\right)^{(-1)^{i}}$.

Our third example is the pants $P$. Contrary to above examples with abelian fundamental groups, $\pi_{1}(P)$ is the free group with two generators. Hence there are irreducible $S U(2)$-representations of $\pi_{1}(P)$. We can see that the $S U(2)$-representation space $R(P, S U(2))$ is the quotient space of $S U(2) \times S U(2)$ by $S O(3)$ since $P$ is homotopy equivalent to the figure eight simplex $P^{\prime}$. We denote the irreducible $S U(2)$-representation space of $\pi_{1}(P)$ by $R(P, S U(2))^{-} \subset R(P, S U(2))$. Since $P$ is 
homotopy equivalent to $P^{\prime}$, we consider a chain complex $C .\left(P^{\prime}, A d(\rho)\right)$ instead of the chain complex $C .(P, A d(\rho))$ for an irreducible $S U(2)$-representation $\rho$

$$
0 \rightarrow C_{1}\left(\tilde{P}^{\prime}\right) \otimes_{\pi} s u(2) \rightarrow C_{0}\left(\tilde{P}^{\prime}\right) \otimes_{\pi} s u(2) \rightarrow 0
$$

where $\tilde{P}^{\prime}$ is the universal covering space of $P^{\prime}$. Then we have

$$
\begin{aligned}
& H_{0}\left(P, s u(2)_{\rho}\right)=H_{2}\left(P, s u(2)_{\rho}\right)=0, \\
& H_{1}\left(P, s u(2)_{\rho}\right)=R\left[x_{1} \otimes v_{1}, x_{2} \otimes v_{2}, x_{3} \otimes v_{3}\right]
\end{aligned}
$$

where $R$ is the real number field, $x_{i}$ 's are the three boundaries of $P$ and $v_{i}$ 's are the $A d\left(\rho\left(x_{i}\right)\right)$ - invariant vectors in $\mathrm{su}(2)$. The R-torsion $\tau(P, A d(\rho))$ is the scalar multiple of

$$
\left(x_{1} \otimes v_{1}\right) \wedge\left(x_{2} \otimes v_{2}\right) \wedge\left(x_{3} \otimes v_{3}\right) \in \operatorname{det} H_{*}\left(P, s u(2)_{\rho}\right)^{-1}
$$

where $\operatorname{det} H_{*}\left(P, s u(2)_{\rho}\right)=\operatorname{det} H_{1}\left(P, s u(2)_{\rho}\right)$ because the representation $\rho$ is irreducible.

In fact $\tau(P, A d(\rho))$ is a volume form of $R(P, S U(2))^{-}$. Now we consider more closely the volume form $\tau(P, A d(\rho))$. Let

$$
\sigma: R(P, S U(2))^{-} \rightarrow \mathcal{L}_{1} \times \mathcal{L}_{2} \times \mathcal{L}_{3}
$$

be the map induced by the restriction from $P$ to $\partial P=\left\{x_{1}, x_{2}, x_{3}\right\}$ where $\mathcal{L}_{i}$ is the $S U(2)$-representation space for a circle $x_{i}$ defined by the same way as $\mathcal{L}$ for $S^{1}$. Recall that $\mathcal{L}_{i}$ can be identified with the maximal torus $T^{1}$ of $S U(2)$. By the definition, $\sigma$ takes the conjugacy class of an irreducible representation $\rho$ to the holonomies of $\rho\left(x_{1}\right), \rho\left(x_{2}\right), \rho\left(x_{3}\right)$. Since $\sigma$ is injective map, there exists an object on $\mathcal{L}_{1} \times \mathcal{L}_{2} \times \mathcal{L}_{3}$ whose pull back is the volume form $\tau(P, \operatorname{Ad}(\rho))$. We denote this by $\sigma_{*} \tau(P, A d(\rho))$. Since we can identify $\mathcal{L}_{i}$ with the maximal torus $T^{1}$, we have that

$$
\sigma_{*} \tau(P, A d(\rho))=f \nu_{1} \nu_{2} \nu_{3}
$$

for some $f \in L^{2}\left(\mathcal{L}_{1} \times \mathcal{L}_{2} \times \mathcal{L}_{3}\right)$ where $\nu_{i}$ is a natural volume form on the maximal torus $T^{1}$ with $\int_{T^{1}} \nu_{i}=1$. From now on $\operatorname{Vol}(G)$ is the volume of the compact Lie group $G$. Then we have the following formula

$$
\sigma_{*} \tau(P, A d(\rho))=\frac{2}{\operatorname{Vol}(S U(2))^{2}} \sum_{\alpha} \frac{1}{n_{\alpha}} \prod_{i=1}^{3} \chi_{\alpha}\left(\rho\left(x_{i}\right)\right) \nu_{1} \nu_{2} \nu_{3}
$$

where the above sum is taken over all the irreducible representations of $S U(2)$, $n_{\alpha}$ is the dimension of the representation space of an irreducible representation $\alpha$. Note that the equality in $(2.4)$ holds in $L^{2}$-sense. The proof of $(2.4)$ is given in [W2].

If we assume that one boundary $x_{3}$ of $P$ has a fixed holonomy, we must modify (2.4). The following formula for a boundary with the fixed holonomy is also used in [W2] without the explicit derivation. So we derive it here. In the following proposition we use the character theory of $S U(2)$. For the detailed fact, see $[\mathrm{B}, \mathrm{tD}]$. 
Proposition 2.5. If the trace of $\rho\left(x_{3}\right)$ is fixed so that $\operatorname{tr}\left(\rho\left(x_{3}\right)\right)=2 \cos (\theta)$ for some fixed $\theta$, then $\sigma_{*} \tau(P, A d(\rho))$ is

$$
\frac{2 \operatorname{Vol}\left(S^{2}\right)}{\operatorname{Vol}(S U(2))^{2}} \sum_{\alpha} \frac{1}{n_{\alpha}} \frac{\sin \left(n_{\alpha} \theta\right)}{\sin (\theta)} \chi_{\alpha}\left(\rho\left(x_{1}\right)\right) \chi_{\alpha}\left(\rho\left(x_{2}\right)\right) \nu_{1} \nu_{2}
$$

where the above sum is taken over all the irreducible representations of $S U(2)$ and $n_{\alpha}$ is the dimension of the representation space of an irreducible representation $\alpha$. $\operatorname{Vol}\left(S^{2}\right)$ is the volume of $S^{2}$ induced from a volume form of $S U(2)$.

Proof. We can consider the set of conjugacy classes of representations satisfying the given condition as the inverse image of the following map

$$
p_{3} \circ \sigma: R(P, S U(2))^{-} \rightarrow \mathcal{L}_{1} \times \mathcal{L}_{2} \times \mathcal{L}_{3} \rightarrow \mathcal{L}_{3}
$$

for $e^{(2 \pi i \theta)} \in \mathcal{L}_{3}$ where $p_{3}$ is the natural projection. We denote this inverse image by $R(P, S U(2), \theta)^{-}$. And let $S^{2}(\theta)$ be the subset of $S U(2)$ with fixed trace $2 \cos (\theta)$. Then there exists the natural projection map $p: S U(2) \times S^{2}(\theta) \rightarrow R(P, S U(2), \theta)^{-}$ with a fiber $S U(2) / Z_{2}$. As above we have a map

$$
\sigma^{\prime}: R(P, S U(2), \theta)^{-} \rightarrow \mathcal{L}_{1} \times \mathcal{L}_{2}
$$

which sends $[\rho]$ in $R(P, S U(2), \theta)^{-}$to the holonomies $\rho\left(x_{1}\right), \rho\left(x_{2}\right)$.

We push $\tau(P, A d(\rho))$ by $\sigma^{\prime}$ so that we get a volume form $\sigma_{*}^{\prime} \tau(P, A d(\rho))$ on $\mathcal{L}_{1} \times \mathcal{L}_{2}$. Then this volume can be written by $f \nu_{1} \nu_{2}$ for some $f \in L^{2}\left(\mathcal{L}_{1} \times \mathcal{L}_{2}\right)$. To find $f$ exactly, we integrate the character $\chi_{\alpha}$ of $\rho\left(x_{1}\right), \rho\left(x_{2}\right)$ for an the irreducible representation $\alpha$ of $S U(2)$. This is because the set of characters $\left\{\chi_{\alpha}\right\}$ for all the irreducible representations of $S U(2)$ is the uniformly dense subset of the continuous function space of $\mathcal{L}_{1} \times \mathcal{L}_{2}$. So we have the following equalities;

$$
\begin{aligned}
W_{\alpha_{1}, \alpha_{2}} & =\int_{\mathcal{L}_{1} \times \mathcal{L}_{2}} \chi_{\alpha_{1}}\left(\rho\left(x_{1}\right)\right) \chi_{\alpha_{2}}\left(\rho\left(x_{2}\right)\right) \sigma_{*}^{\prime} \tau(P, A d(\rho)) \\
& =\frac{2}{\operatorname{Vol}(S U(2))} \int_{S U(2) \times S^{2}(\theta)} p^{*}{\sigma^{\prime *}}^{*}\left(\chi_{\alpha_{1}}\left(\rho\left(x_{1}\right)\right) \chi_{\alpha_{2}}\left(\rho\left(x_{2}\right)\right)\right) d U_{2} d g(\theta) \\
& =\frac{2}{\operatorname{Vol}(S U(2))} \int_{S U(2) \times S^{2}(\theta)} \chi_{\alpha_{1}}\left(g(\theta)^{-1} \rho\left(x_{2}\right)^{-1}\right) \chi_{\alpha_{2}}\left(\rho\left(x_{2}\right)\right) d U_{2} d g(\theta) \\
& =2 \frac{\delta_{\alpha_{1}, \alpha_{2}}}{n_{\alpha_{1}}} \int_{S^{2}(\theta)} \chi_{\alpha_{1}}\left(g(\theta)^{-1}\right) d g(\theta) \\
& =2 \delta_{\alpha_{1}, \alpha_{2}} \frac{\chi_{\alpha_{1}}\left(g(\theta)^{-1}\right)}{n_{\alpha_{1}}} \operatorname{Vol}\left(S^{2}(\theta)\right) .
\end{aligned}
$$


So we have that

$$
\sigma_{*}^{\prime} \tau(P, A d(\rho))=\frac{2 \operatorname{Vol}\left(S^{2}(\theta)\right)}{\operatorname{Vol}(S U(2))^{2}} \sum_{\alpha} \frac{1}{n_{\alpha}} \chi_{\alpha}\left(g(\theta)^{-1}\right) \chi_{\alpha}\left(\rho\left(x_{1}\right)\right) \chi_{\alpha}\left(\rho\left(x_{2}\right)\right) \nu_{1} \nu_{2} .
$$

On the other hand, $\chi_{\alpha}\left(g(\theta)^{-1}\right)=\frac{\sin \left(n_{\alpha} \theta\right)}{\sin (\theta)}$ and $\operatorname{Vol}\left(S^{2}(\theta)\right)=\operatorname{Vol}\left(S^{2}\right)$. So we get the formula in the proposition.

\section{$\S 3$ R-torsion of Seifert fibred manifolds and graph manifolds}

In this section we compute the scalar part of the R-torsion of Seifert fibred manifolds and graph manifolds for the Ad-SU(2)-representation. This quantity gives the weight of the half density for each connected component of the irreducible SU(2)-representation space of Seifert fibred manifold or graph manifold. Our computing method in this section comes from $[\mathrm{F}]$.

First we consider the manifolds whose R-torsion we will compute. We denote the Seifert fibred manifold with the Seifert invariant $\left\{g ;\left(\alpha_{1}, \beta_{1}\right), \ldots,\left(\alpha_{m}, \beta_{m}\right)\right\}$ by $M=M\left(g ;\left(\alpha_{1}, \beta_{1}\right), \ldots,\left(\alpha_{m}, \beta_{m}\right)\right)$. We have that $\pi_{1}(M)$ is

$$
\left\{a_{i}, b_{i}, q_{j}, h:\left[h, a_{i}\right]=\left[h, b_{i}\right]=\left[h, q_{j}\right]=1, q_{j}^{\alpha_{j}} h^{\beta_{j}}=1, \prod_{j=1}^{m} q_{j} \prod_{i=1}^{g}\left[a_{i}, b_{i}\right]=1\right\}
$$

We assume that $g \geqq 2$ from now on. The irreducible $S U(2)$-representation of $\pi_{1}(M)$ is well known. See $[\mathrm{A}],[\mathrm{F}, \mathrm{S}],[\mathrm{K}, \mathrm{K}]$ for the irreducible $S U(2)$-representation of $\pi_{1}(M)$. We review some facts that we need.

Since $h$ is central in $\pi_{1}(M)$, an irreducible representation $\rho$ takes $h$ to \pm 1 in $S U(2)$. So the trace of $\rho\left(q_{j}\right)$ is $2 \cos \left(\frac{\pi n_{j}}{\alpha_{j}}\right)$. The set of numbers $\left\{n_{1}, \ldots, n_{m}\right\}$ which are called as 'rotation numbers' determines a connected component of the irreducible $S U(2)$-representation space $R(M, S U(2))^{-}$. The rotation number $n_{j}$ is even(odd) if $\beta_{j}$ is even(odd).

Next we consider a graph manifold which is made from two Seifert fibred manifolds $M_{1}, M_{2}$ which have a torus boundary each. We review some materials about graph manifolds of $[\mathrm{K}, \mathrm{K}] . M_{1}$ is given by deleting a solid torus $D^{2} \times S^{1}$ from $M$ where a disk $D^{2}$ lies in the base surface $\Sigma_{g}$ and a circle $S^{1}$ is the fiber component of the Seifert fibration. Then $\pi_{1}\left(M_{1}\right)$ is

$$
\pi_{1}\left(M_{1}\right)=\left\{a_{i}, b_{i}, q_{j}, h:\left[h, a_{i}\right]=\left[h, b_{i}\right]=\left[h, q_{j}\right]=1, q_{j}^{\alpha_{j}} h^{\beta_{j}}=1\right\} .
$$


The other manifold $M_{2}$ can be constructed by the same way from the another Seifert manifold $M\left(g^{\prime} ;\left(\alpha_{1}^{\prime}, \beta_{1}^{\prime}\right), \ldots,\left(\alpha_{n}^{\prime}, \beta_{n}^{\prime}\right)\right)$. Then $\pi_{1}\left(M_{2}\right)$ is

$$
\pi_{1}\left(M_{2}\right)=\left\{a_{i^{\prime}}^{\prime}, b_{i^{\prime}}^{\prime}, r_{j^{\prime}}, k:\left[k, a_{i^{\prime}}^{\prime}\right]=\left[k, b_{i^{\prime}}^{\prime}\right]=\left[k, r_{j^{\prime}}\right]=1, r_{j^{\prime}}^{\alpha_{j^{\prime}}^{\prime}} k^{\beta_{j^{\prime}}^{\prime}}=1\right\} .
$$

Since $\partial M_{1}=\partial M_{2}=T^{2}$, we can glue two manifolds $M_{1}, M_{2}$ by an automorphism $\phi$ of $T^{2}$. We assume that the meridian, the longitude pairs of $\partial M_{1}, \partial M_{2}$ is given by

$$
\begin{aligned}
& \left\{\mu_{1}, \lambda_{1}\right\}=\left\{\prod_{j=1}^{m} q_{j} \prod_{i=1}^{g}\left[a_{i}, b_{i}\right], h\right\}, \\
& \left\{\mu_{2}, \lambda_{2}\right\}=\left\{\prod_{j^{\prime}=1}^{n} r_{j^{\prime}} \prod_{i^{\prime}=1}^{g^{\prime}}\left[a_{i^{\prime}}^{\prime}, b_{i^{\prime}}^{\prime}\right], k\right\} .
\end{aligned}
$$

Define $\phi: \partial M_{1} \rightarrow \partial M_{2}$ by

$$
\begin{aligned}
& \phi\left(\mu_{1}\right)=\alpha \mu_{2}+\beta \lambda_{2}, \\
& \phi\left(\lambda_{1}\right)=\gamma \mu_{2}+\delta \lambda_{2}
\end{aligned}
$$

where $\alpha \delta-\beta \gamma=-1$. Then we have a glued manifold $N=M_{1} \cup_{\phi} M_{2}$ and we call this manifold as graph manifold. Later we need distinguish two cases when $\gamma=0, \gamma \neq 0$. When we need clarify the dependence of $\gamma$, we denote graph manifold by $N_{\gamma}$ to express the dependence of $\gamma$. The natural question is how we glue the irreducible $S U(2)$-representations $\rho_{1}, \rho_{2}$ of the manifolds $M_{1}, M_{2}$ to get an irreducible $S U(2)$-representation of graph manifold $N$. In fact, there are a condition of gluing of representations. Since $\pi_{1}\left(\partial M_{i}\right)$ is abelian, we have

$$
\begin{array}{ll}
\rho_{1}\left(\mu_{1}\right)=\left(\begin{array}{cc}
e^{2 \pi i \phi_{1}} & 0 \\
0 & e^{-2 \pi i \phi_{1}}
\end{array}\right), & \rho_{1}\left(\lambda_{1}\right)=\left(\begin{array}{cc}
e^{2 \pi i \psi_{1}} & 0 \\
0 & e^{-2 \pi i \psi_{1}}
\end{array}\right), \\
\rho_{2}\left(\mu_{2}\right)=\left(\begin{array}{cc}
e^{2 \pi i \phi_{2}} & 0 \\
0 & e^{-2 \pi i \phi_{2}}
\end{array}\right), & \rho_{2}\left(\lambda_{2}\right)=\left(\begin{array}{cc}
e^{2 \pi i \psi_{2}} & 0 \\
0 & e^{-2 \pi i \psi_{2}}
\end{array}\right) .
\end{array}
$$

We can see that $\psi_{1}, \psi_{2} \in Z\left[\frac{1}{2}\right]$ since $h, k$ are central. Then the condition that a glued representation $\rho$ exists is that

$$
\phi_{1}=\alpha \phi_{2}+\beta \psi_{2}, \quad \psi_{1}=\gamma \phi_{2}+\delta \psi_{2}
$$

This condition comes from (3.1). 
To compute the R-torsions of Seifert fibred manifolds and graph manifolds we decompose these manifolds into simple pieces which we considered in section 2 . For the Seifert fibred manifold $M\left(g ;\left(\alpha_{1}, \beta_{1}\right), \ldots,\left(\alpha_{m}, \beta_{m}\right)\right)$, we can decompose $M$ into

$m$-solid tori $A_{j}=D_{j}^{2} \times S^{1}$ and $(2(g-1)+m)$-copies of $X_{i}=P_{i} \times S^{1}$

where a disk $D_{j}^{2}$ lies the base surface $\Sigma_{g}, S^{1}$ is the fiber component and $P_{i}$ 's are the pairs of pants. The decomposition is the inverse process of construction of $M$ and the pants decomposition of $\Sigma_{g}-\cup_{j=1}^{m} D_{j}^{2}$. We can assume that each $X_{i}$ meets only one $A_{j}$ or does not meet $A_{j}$ by the isotopic moves of the exceptional fibers.

For graph manifolds $N=M_{1} \cup_{\phi} M_{2}$, we decompose $N$ into $M_{1}, M_{2}$ and then apply the same process to $M_{i}$ as above. So we have

$m$-solid tori $A_{j}, n$-solid tori $B_{j^{\prime}}$, and $(2 g+m-1)$-copies of $X_{i},\left(2 g^{\prime}+n-1\right)$-copies of $Y_{i^{\prime}}$

where $X_{i}, Y_{i^{\prime}}$ are homeomorphic to $P \times S^{1}$ and

$$
M_{1}=\cup_{i=1}^{2 g+m-1} X_{i} \cup_{j=1}^{m} A_{j} \quad \text { and } \quad M_{2}=\cup_{i^{\prime}=1}^{2 g^{\prime}+n-1} Y_{i^{\prime}} \cup_{j^{\prime}=1}^{n} B_{j^{\prime}} .
$$

We can assume that $X_{1}$ meets $M_{2}, Y_{1}$ meets $M_{1}$ and that $X_{i}\left(Y_{i^{\prime}}\right)$ meets only one $A_{j}\left(B_{j^{\prime}}\right)$ or does not meet $A_{j}\left(B_{j^{\prime}}\right)$ as above.

We consider $S U(2)$-representations of $\pi_{1}(M)$ or $\pi_{1}(N)$ such that the induced representations by restriction to each pieces $X_{i}$ or $X_{i}, Y_{i^{\prime}}$ are irreducible. We call such a representation as totally irreducible representation for $M$ or $N$ with respect to above fixed decomposition.

As the previous section we denote the highest wedge product of a basis of $H_{i}\left(X, s u(2)_{\rho_{X}}\right)$ by $D_{H_{i}\left(X, s u(2)_{\rho_{X}}\right)}$ and $\otimes_{i=0}^{d}\left(D_{H_{i}\left(X, s u(2)_{\rho_{X}}\right)}\right)^{(-1)^{i}}$ by $D_{H_{*}(X)}$ for a manifold $X$ of $d$-dimension. We denote the dimension of $H_{i}\left(X, s u(2)_{\rho_{X}}\right)$ by $h_{i}(X)$ in the following proposition. 
Proposition 3.3. Let $\rho_{M}, \rho_{N_{\gamma}}$ be irreducible $S U(2)$-representations of $\pi_{1}(M)$, $\pi_{1}\left(N_{\gamma}\right)$ with rotation numbers $\left\{n_{j}\right\},\left\{n_{j}, n_{j^{\prime}}^{\prime}\right\}$. We assume that $\rho_{M}, \rho_{N_{\gamma}}$ are totally irreducible for $M, N_{\gamma}$. The R-torsion of the Seifert fibred manifold $M\left(g ;\left(\alpha_{j}, \beta_{j}\right)\right)$ and the graph manifold $N_{\gamma}=M_{1}\left(g ;\left(\alpha_{j}, \beta_{j}\right)\right) \cup_{\phi_{\gamma}} M_{2}\left(g^{\prime} ;\left(\alpha_{j^{\prime}}^{\prime}, \beta_{j^{\prime}}^{\prime}\right)\right)$ is given by

$$
\begin{aligned}
\tau\left(M, A d\left(\rho_{M}\right)\right) & =4^{m} \prod_{j=1}^{m} \frac{\sin ^{2}\left(\frac{\pi n_{j} \beta_{j}^{*}}{\alpha_{j}}\right)}{\alpha_{j}} D_{H_{*}(M)}^{-1} \in \operatorname{det} H_{*}\left(M, s u(2)_{\rho_{M}}\right)^{-1}, \\
\tau\left(N_{\gamma}, A d\left(\rho_{N_{\gamma}}\right)\right) & =4^{m+n} f(\gamma) \prod_{j=1}^{m} \frac{\sin ^{2}\left(\frac{\pi n_{j} \beta_{j}^{*}}{\alpha_{j}}\right)}{\alpha_{j}} \prod_{j^{\prime}=1}^{n} \frac{\sin ^{2}\left(\frac{\left.\pi n_{j^{\prime} \beta^{\prime}{ }_{j}^{*}}^{\alpha_{j^{\prime}}^{\prime}}\right)}{\alpha_{j^{\prime}}^{\prime}} D_{H_{*}\left(N_{\gamma}\right)}^{-1}\right.}{} \\
& \in \operatorname{det} H_{*}\left(N_{\gamma}, \operatorname{su}(2)_{\rho_{N}}\right)^{-1}
\end{aligned}
$$

where

$$
\begin{aligned}
& \beta_{j} \beta_{j}^{*}=1 \quad \bmod \alpha_{j}, \quad \beta_{j^{\prime}}^{\prime} \beta_{j^{\prime}}^{\prime}=1 \bmod \alpha_{j^{\prime}}^{\prime}, \\
& f(\gamma)=\left\{\begin{array}{l}
1 \quad \text { if } \gamma=0 \\
\frac{1}{|\gamma|} \text { if } \gamma \neq 0
\end{array}\right.
\end{aligned}
$$

$h_{1}(M)=h_{2}(M)$ is $6(g-1)+2 m$ and $h_{1}\left(N_{\gamma}\right)=h_{2}\left(N_{\gamma}\right)$ is $6\left(g+g^{\prime}-1\right)+2(m+n)$, $6\left(g+g^{\prime}\right)+2(m+n)-7$ if $\gamma=0, \gamma \neq 0$.

Before we prove the proposition, we remark some facts. We can see that the R-torsion depends on the gluing torus automorphisms of the decomposed pieces by $\left(\alpha_{j}, \beta_{j}\right),\left(\alpha_{j^{\prime}}^{\prime}, \beta_{j^{\prime}}^{\prime}\right)$ and the representation $\rho_{M}, \rho_{N}$ by rotation numbers $\left(n_{j}\right),\left(n_{j}, n_{j^{\prime}}^{\prime}\right)$. Since Ad-SU(2)-representation is unimodular, the highest wedge product of $D_{H_{i}\left(M, s u(2)_{M}\right)}$ does not depend on the basis change of $C_{i}(\tilde{M})$ when a basis of $s u(2)$ is fixed. In the above proposition we have that $D_{H_{*}(\cdot)}^{-1}=D_{H_{1}(\cdot, s u(2))} \otimes$ $D_{H_{2}(\cdot, s u(2))}^{-1}$ since we assume that the representations are irreducible.

Proof. We shall compute the R-torsion of graph manifold $N_{\gamma}$ for the totally irreducible $S U(2)$-representation with respect to the given decomposition. For Seifert fibred manifolds we get the result in the process of computation of graph manifold.

First we define some tori from the decomposed pieces such that

$$
\begin{aligned}
T_{j} & =\partial A_{j}, \quad T_{j^{\prime}}^{\prime}=\partial B_{j^{\prime}}, \\
T_{i_{1} i_{2}} & =X_{i_{1}} \cap X_{i_{2}}, \quad T_{i_{1}^{\prime} i_{2}^{\prime}}^{\prime}=Y_{i_{1}^{\prime}} \cap Y_{i_{2}^{\prime}}, \quad T=M_{1} \cap M_{2} .
\end{aligned}
$$

We have a following formula about the R-torsions of each pieces and the given manifold $N_{\gamma}$ such that 


$$
\begin{aligned}
& \tau\left(N_{\gamma}\right)=\otimes_{\left\{i, i^{\prime}, j, j^{\prime}\right\}} \tau\left(X_{i}\right) \cdot \tau\left(A_{j}\right) \cdot \tau\left(Y_{i^{\prime}}\right) \cdot \tau\left(B_{j^{\prime}}\right) \\
& \quad\left(\otimes_{\left\{i, i^{\prime}, j, j^{\prime}\right\}} \tau\left(T_{j}\right) \cdot \tau\left(T_{j^{\prime}}^{\prime}\right) \cdot \tau\left(T_{i_{1} i_{2}}\right) \cdot \tau\left(T_{i_{1}^{\prime} i_{2}^{\prime}}^{\prime}\right) \cdot \tau(T)\right)^{-1} \otimes_{r=1}^{\infty} \tau\left(E_{.}^{r}\right)^{-1}
\end{aligned}
$$

where - denotes the tensor product and the last term $\tau\left(E^{r}\right)$ is the torsion of the spectral sequence of the generalized Meyer-Vietoris sequence. For the proof of (3.4), see [F]. In the above formula we omit the representation notation for convenience.

Since $A_{j}, B_{j^{\prime}}$ are homotopy equivalent to $S^{1}$, we can consider $S^{1}$ instead of $A_{j}, B_{j^{\prime}}$ for the R-torsion. Let $e$ be 0 -cell of each pieces, $a_{j}, b_{j^{\prime}}$ be 1 -cells of $S^{1}$ 's which are homotopy equivalent to $A_{j}, B_{j^{\prime}}$. Then we have that $D_{H_{*}\left(A_{j}\right)}=$ $e \otimes a_{j}^{-1}$ and $D_{H_{*}\left(B_{j^{\prime}}\right)}=e \otimes b_{j^{\prime}}^{-1}$. We omit the notation of invariant vectors of $\operatorname{Ad}(\rho)\left(a_{j}\right), \operatorname{Ad}(\rho)\left(b_{j^{\prime}}\right)$ for convenience. Then we have following formulas from the construction of $N_{\gamma}$ and (2.1)

$$
\begin{aligned}
\tau\left(A_{j}\right) & =4 \sin ^{2}\left(\frac{\pi n_{j} \gamma_{j}}{\alpha_{j}}\right) D_{H_{*}\left(A_{j}\right)}^{-1} \\
\tau\left(B_{j^{\prime}}\right) & =4 \sin ^{2}\left(\frac{\pi n_{j^{\prime}}^{\prime} \gamma_{j^{\prime}}^{\prime}}{\alpha_{j^{\prime}}^{\prime}}\right) D_{H_{*}\left(B_{j^{\prime}}\right)}^{-1}
\end{aligned}
$$

where $\beta_{j} \gamma_{j}=1 \bmod \alpha_{j}, \beta_{j^{\prime}}^{\prime} \gamma_{j^{\prime}}^{\prime}=1 \bmod \alpha_{j^{\prime}}^{\prime}$ and $\gamma_{j}, \gamma_{j^{\prime}}^{\prime}$ are given by the twists of the exceptional fibers.

For $X_{i}, Y_{i^{\prime}}$ which are homeomorphic to $P_{i} \times S^{1}, P_{i^{\prime}}^{\prime} \times S^{1}$, we can see that the $S^{1}$-component has the trivial holonomy since the given representations take $h, k$ into $\pm 1 \in S U(2)$. Hence we have that

$$
\begin{aligned}
\tau\left(X_{i}\right) & =\left(x_{i}^{1} \otimes x_{i}^{2} \otimes x_{i}^{3}\right) \otimes\left(\left(x_{i}^{1} \cup h\right) \otimes\left(x_{i}^{2} \cup h\right) \otimes\left(x_{i}^{3} \cup h\right)\right)^{-1} \\
& =D_{H_{*}\left(X_{i}\right)}^{-1} \quad, \\
\tau\left(Y_{i^{\prime}}\right) & =\left(y_{i^{\prime}}^{1} \otimes y_{i^{\prime}}^{2} \otimes y_{i^{\prime}}^{3}\right) \otimes\left(\left(y_{i^{\prime}}^{1} \cup k\right) \otimes\left(y_{i^{\prime}}^{2} \cup k\right) \otimes\left(y_{i^{\prime}}^{3} \cup k\right)\right)^{-1} \\
& =D_{H_{*}\left(Y_{i^{\prime}}\right)}^{-1}
\end{aligned}
$$

where $\partial P_{i}=\left\{x_{i}^{1}, x_{i}^{2}, x_{i}^{3}\right\}$ and $\partial P_{i^{\prime}}^{\prime}=\left\{y_{i^{\prime}}^{1}, y_{i^{\prime}}^{2}, y_{i^{\prime}}^{3}\right\}$. We omit the notation of invariant vectors under $A d\left(\rho\left(x_{i}^{\prime}\right)\right), A d\left(\rho\left({\dot{j^{\prime}}}^{\prime}\right)\right)$ for convenience. For the tori $T_{i}, T_{i^{\prime}}^{\prime}, T_{i, i . .}$, $T_{i^{\prime} i^{\prime} .}^{\prime}, T$, we have the value of R-torsion for these tori from (2.2). 
Now we compute the spectral sequence terms $E_{\text {. }}^{r}$ First $E^{1}$ is given by

$$
\begin{aligned}
& 0 \quad \longleftarrow \quad \oplus_{i=1}^{l_{1}} H_{2}\left(Z_{i}\right) \quad \stackrel{\delta_{2}}{\longleftarrow} \oplus_{i=1}^{l_{2}} H_{2}\left(T_{i}^{2}\right) \longleftarrow 0 \\
& 0 \longleftarrow \oplus_{i=1}^{l_{1}} H_{1}\left(Z_{i}\right) \oplus_{i=1}^{m+n} H_{1}\left(C_{i}\right) \stackrel{\delta_{1}}{\longleftarrow} \oplus_{i=1}^{l_{2}} H_{1}\left(T_{i}^{2}\right) \longleftarrow 0 \\
& 0 \longleftarrow \oplus_{i=1}^{m+n} H_{0}\left(C_{i}\right) \quad \longleftarrow \quad \delta_{0} \quad \oplus_{i=1}^{l_{2}} H_{0}\left(T_{i}^{2}\right) \longleftarrow 0
\end{aligned}
$$

where $l_{1}=2\left(g+g^{\prime}-1\right)+(m+n), l_{2}=3\left(g+g^{\prime}-1\right)+2(m+n)$ and

$$
\begin{aligned}
& \cup_{i}^{l_{1}} Z_{i}=\cup_{i}^{2 g-1+m} X_{i} \cup_{i^{\prime}}^{2 g^{\prime}-1+n} Y_{i^{\prime}}, \\
& \cup_{i}^{m+n} C_{i}=\cup_{j}^{m} A_{j} \cup_{j^{\prime}}^{n} B_{j^{\prime}} \text {, } \\
& \cup_{i=1}^{l_{2}} T_{i}^{2}=\cup_{j=1}^{m} T_{j} \cup_{j^{\prime}=1}^{n} T_{j^{\prime}}^{\prime} \cup_{\{i . i . .\}} T_{i . i . .} \cup_{\left\{i^{\prime} i_{i}^{\prime} .\right\}} T_{i^{\prime} i^{\prime} .}^{\prime} \cup T \text {. }
\end{aligned}
$$

We can see that

$$
\begin{array}{ll}
\delta_{0}(e)=e \quad \text { for } & e \in \oplus_{i} H_{0}\left(T_{i}\right) \oplus_{i^{\prime}} H_{0}\left(T_{i^{\prime}}^{\prime}\right), \\
\delta_{0}(e)=0 & \text { for } \quad e \in \oplus_{\{i . i . .\}} H_{0}\left(T_{i . i . .}\right) \oplus_{\left\{i^{\prime} i^{\prime} . .\right\}} H_{0}\left(T_{i^{\prime} i^{\prime} . .}^{\prime}\right) \oplus H_{0}(T) .
\end{array}
$$

Hence the dimension of kernel of $\delta_{0}$ is $3\left(g+g^{\prime}-1\right)+(m+n)$.

Since every gluing is made from the torus automorphism, we have that

$$
\delta_{2}(l \cup m)=l \cup m \quad \text { for a basis of } \quad l \cup m \in \oplus_{i}^{l_{2}} H_{2}\left(T_{i}^{2}\right) .
$$

Hence the dimension of cokernel of $\delta_{2}$ is $3\left(g+g^{\prime}-1\right)+(m+n)$.

The map $\delta_{1}$ depends on the gluing-torus automorphism more explicitly. To describe this map we introduce a natural basis of $\oplus_{i=1}^{l_{2}} H_{1}\left(T_{i}^{2}\right)$ such that

$$
\begin{aligned}
& H_{1}\left(T_{j}\right)=R\left[l_{j}, m_{j}\right], \quad H_{1}\left(T_{j^{\prime}}^{\prime}\right)=R\left[l_{j^{\prime}}^{\prime}, m_{j^{\prime}}^{\prime}\right], \\
& H_{1}\left(T_{i . i . .}\right)=R\left[l_{i . i_{. .}}, m_{i . i . .}\right], \quad H_{1}\left(T_{i^{\prime} i^{\prime} .}^{\prime}\right)=R\left[l_{i^{\prime} i_{. .}^{\prime}}^{\prime}, m_{i^{\prime} i^{\prime} . .}^{\prime}\right. \\
& H_{1}(T)=R[l, m]
\end{aligned}
$$

where $R$ is the real number field. Then we have that

$$
\begin{aligned}
& \delta_{1}\left(m_{j}\right)=\alpha_{j} x_{i} \oplus 0 \in H_{1}\left(X_{i}\right) \oplus H_{1}\left(A_{j}\right), \\
& \delta_{1}\left(l_{j}\right)=\gamma_{j} x_{i} \oplus a_{j} \in H_{1}\left(X_{i}\right) \oplus H_{1}\left(A_{j}\right) \text { for some } i
\end{aligned}
$$

where $i$ is given such that $A_{j}$ meets $X_{i}$ and $\gamma_{j}$ is given by the gluing torus automorphism as (3.5). For other intersection tori we have that

$$
\begin{aligned}
& \delta_{1}\left(m_{i . i .}\right)=x_{i .} \oplus x_{i . .} \in H_{1}\left(X_{i .}\right) \oplus H_{1}\left(X_{i . .}\right), \\
& \delta_{1}\left(l_{i . i . .}\right)=0 \in H_{1}\left(X_{i .}\right) \oplus H_{1}\left(X_{i . .}\right) .
\end{aligned}
$$


similar formulas hold in the gluing of the part of $M_{2}$. And finally we have that

$$
\begin{aligned}
& \delta_{1}(m)=\dot{x_{1}} \oplus \alpha \dot{y_{1}} \in H_{1}\left(X_{1}\right) \oplus H_{1}\left(Y_{1}\right), \\
& \delta_{1}(l)=0 \oplus \gamma y_{1} \in H_{1}\left(X_{1}\right) \oplus H_{1}\left(Y_{1}\right) .
\end{aligned}
$$

The kernel of $\delta_{1}$ is generated by

$$
\begin{aligned}
& \left\{l_{i . i . .}, l_{i^{\prime} i^{\prime} .}^{\prime}\right\} \quad \text { if } \quad \gamma \neq 0 \\
& \left\{l_{i . i . .}, l_{i^{\prime} i^{\prime} .}^{\prime}, l\right\} \quad \text { if } \quad \gamma=0 .
\end{aligned}
$$

Hence the dimension of the kernel of $\delta_{1}$ which is same as the dimension of the cokernel of $\delta_{1}$ is $3\left(g+g^{\prime}-1\right)+(m+n)$ or $3\left(g+g^{\prime}\right)+(m+n)-4$ if $\gamma=0$ or $\gamma \neq 0$.

From above we can see how $E^{2}$ is given. In fact $E^{2}$. is the $H_{*}(N)$. If we gather $(3.5) \sim(3.10)$ and apply these to (3.4), then we get the result about R-torsion in the proposition. Moreover we can see that $h_{1}\left(N_{\gamma}\right)$ is the sum of the dimension of the kernel of $\delta_{0}$ and the dimension of the cokernel of $\delta_{1}$ and that $h_{2}\left(N_{\gamma}\right)$ is the sum of the dimension of the kernel of $\delta_{1}$ and the dimension of the cokernel of $\delta_{2}$ in $E^{1}$.

\section{$\S 4$ Half Density Volumes of Representation Spaces}

In this section we compute the half density volumes of the irreducible $S U(2)$ representation spaces of Seifert fibred manifold $M$ and graph manifold $N$. The half density volume comes from the R-torsion, more precisely from the determinant term of the first homology in the R-torsion. The tangent space of $R(M, S U(2))^{-}$at $\left[\rho_{M}\right]$ can be identified with the first cohomology $H^{1}\left(M, s u(2)_{\rho_{M}}\right)$. So the determinant of the first homology $D_{H_{1}\left(M, s u(2)_{\rho_{M}}\right)}$ gives a volume form of $R(M, S U(2))^{-}$.

As we studied in the section 3 , the sets of the rotation numbers $\left\{n_{j}\right\},\left\{n_{j}, n_{j^{\prime}}^{\prime}\right\}$ of the $S U(2)$-representations $\rho_{M}, \rho_{N}$ determine a connected component of the irreducible $S U(2)$-representation spaces $R(M, S U(2))^{-}, R(N, S U(2))^{-}$. We denote the connected component of irreducible $S U(2)$-representation spaces determined by $\left\{n_{j}\right\},\left\{n_{j}, n_{j^{\prime}}^{\prime}\right\}$ by $R\left(M,\left(n_{j}\right)\right), R\left(N,\left(n_{j}, n_{j^{\prime}}^{\prime}\right)\right)$. Hence we have the following equalities about the volume of $R(M, S U(2))^{-}, \operatorname{Vol}\left(R(M, S U(2))^{-}\right)$for the Seifert fibred manifold $M$; 


$$
\begin{aligned}
\operatorname{Vol}\left(R(M, S U(2))^{-}\right) & =\int_{R(M, S U(2))^{-}} \tau\left(M, A d\left(\rho_{M}\right)\right)^{\frac{1}{2}} \\
& =\sum_{\left\{n_{j}\right\}} \int_{R\left(M,\left(n_{j}\right)\right)} \tau\left(M, A d\left(\rho_{M}\right)\right)^{\frac{1}{2}} \\
& =2^{m} \sum_{\left\{n_{j}\right\}} \prod_{j=1}^{m} \frac{\left|\sin \left(\frac{2 \pi n_{j} \beta_{j}^{*}}{\alpha_{j}}\right)\right|}{\left|\alpha_{j}\right|^{\frac{1}{2}}} \int_{R\left(M,\left(n_{j}\right)\right)} D_{H_{1}\left(M, s u(2)_{\rho_{M}}\right)} .
\end{aligned}
$$

The similar formula holds for the graph manifold $N$. Hence we restrict our concern to the connected component $R\left(M,\left(n_{j}\right)\right), R\left(N,\left(n_{j}, n_{j^{\prime}}^{\prime}\right)\right)$.

Before we compute the term $\int_{R\left(M,\left(n_{j}\right)\right)} D_{H_{1}\left(M, s u(2)_{\rho_{M}}\right)}$, we remark some facts. The spaces over which we integrate are non-compact open manifolds. We will use only the totally irreducible representations in the process of the integration to get the half density volume of the representation space. This is possible because the complement of the totally irreducible representations are lower dimensional so that the complement can be considered as the measure zero set. Hence we can get the volume if we use only the totally irreducible representations with respect to the given decomposition in the section 3 .

We shall use the generic fibration structure of $R\left(M,\left(n_{j}\right)\right), R\left(N,\left(n_{j}, n_{j^{\prime}}^{\prime}\right)\right)$ in order to integrate the half density volume form. So we introduce the generic fibration structures of $R\left(M,\left(n_{j}\right)\right), R\left(N,\left(n_{j}, n_{j^{\prime}}\right)\right)$.

Recall the base surface $\Sigma_{g}$ of $M$. We delete the m-disks $D_{j}^{2}$ 's in $\Sigma_{g}$ where $\partial D_{j}^{2}$ is free homotopic to $q_{j}$. We denote this by $\Sigma_{g, m}$. From the condition $q_{j}^{\alpha_{j}} h^{\beta_{j}}=1$ we can give the holonomy conditions on $\partial \Sigma_{g, m}$. We denote the space of the irreducible $S U(2)$-representations of $\pi_{1}\left(\Sigma_{g, m}\right)$ satisfying above holonomy conditions by $R\left(\Sigma_{g, m},\left(n_{j}\right)\right)$ where $\left(n_{j}\right)$ can be determined as the rotation numbers which determine the connected component of $R(M, S U(2))^{-}$. In fact, we see easily that $R\left(\Sigma_{g, m},\left(n_{j}\right)\right)$ can be identified with $R\left(M,\left(n_{j}\right)\right)$. So we use this identification from now on.

We decompose $\Sigma_{g, m}$ into the $2(g-1)+2 m$-pairs of pants $P_{i}$ with $3(g-1)+m$ intersection circles $S_{i}^{1}$ between the pair of pants such that $X_{i}=P_{i} \times S^{1}$ for the fiber circle $S^{1}$. We denote the $S U(2)$-representation space over $S_{i}^{1}$ by $\mathcal{L}_{i}$ as the section 2. Then there is a natural map

$$
p: R\left(\Sigma_{g, m},\left(n_{j}\right)\right) \rightarrow \mathcal{L}_{1} \times \cdots \times \mathcal{L}_{3(g-1)+m}
$$


induced by the restriction such that

$$
p([\rho])=\left(\left[\left.\rho\right|_{S_{1}^{1}}\right], \ldots,\left[\left.\rho\right|_{S_{3(g-1)+m}^{1}}\right]\right) .
$$

We consider the $S U(2)$-representation $\rho_{\Sigma_{g, m}}$ of $\pi_{1}\left(\Sigma_{g, m}\right)$ such that the representation $\left.\rho\right|_{P^{i}}$ induced by the restriction to each pieces $P_{i}$ is irreducible. Such a representation of $\pi_{1}\left(\Sigma_{g, m}\right)$ corresponds to a totally irreducible representation of $\pi_{1}(M)$ with respect to the corresponding decomposition of $M$. For such a representation $\rho_{\Sigma_{g, m}}$, the inverse image of $p\left(\rho_{\Sigma_{g, m}}\right)$ can be identified with the $3(g-1)+m$ copies of the maximal torus $T^{1}$ divided by $(2 g+m-3)$ copies of the center $Z_{2}$ of $S U(2)$. The maximal torus $T^{1}$ gives the gluing data between the pair of pants and the center $Z_{2}$ comes from some symmetry. For the detail of the proof about this fact, see the proposition 3.8 of [J,W 2], (4.65) of [W2]. So we have a fibration structure of $R\left(\Sigma_{g, m},\left(n_{j}\right)\right)=R\left(M,\left(n_{j}\right)\right)$ generically such that

$$
0 \rightarrow \oplus_{i=1}^{3(g-1)+m} T_{i}^{1} / \oplus_{i=1}^{2 g+m-3} Z_{2_{i}} \rightarrow R\left(M,\left(n_{j}\right)\right) \rightarrow \oplus_{i=1}^{3(g-1)+m} \mathcal{L}_{i} \rightarrow 0 .
$$

The 'generic fibration' means that the fibration exists only for the totally irreducible representations.

By the similar way we can consider a generic fibration for the graph manifold $N_{\gamma}$. But we need distinguish the cases when $\gamma=0$ and $\gamma \neq 0$ for graph manifolds. We recall $(3.2)$

$$
\phi_{1}=\alpha \phi_{2}+\beta \psi_{2}, \quad \psi_{1}=\gamma \phi_{2}+\delta \psi_{2}
$$

where $\psi_{i}$ is fixed for each connected component $R\left(N,\left(n_{j}, n_{j^{\prime}}^{\prime}\right)\right)$. If $\gamma=0$, we can see that

$$
\psi_{1}= \pm \psi_{2} \quad \text { and } \quad \phi_{1}= \pm \phi_{2}+\beta \psi_{2}
$$

since $\alpha \delta=-1$. So the variables $\phi_{1}, \phi_{2}$ are related by

$$
\phi_{1}= \pm \phi_{2}+\beta \psi_{2}
$$

If $\gamma \neq 0$, we have that

$$
\phi_{2}=\frac{1}{\gamma} \psi_{1}-\frac{\delta}{\gamma} \psi_{2}
$$

If we combine this with the first equality of (3.2), then we have that

$$
\phi_{1}=\frac{\alpha}{\gamma} \psi_{1}+\frac{1}{\gamma} \psi_{2}
$$


So the variables $\phi_{1}, \phi_{2}$ are fixed.

We can consider the base surface $\Sigma_{g, 1}$ of $M_{1}$ and the base surface $\Sigma_{g^{\prime}, 1}$ of $M_{2}$. We delete $m$-disks, $n$-disks from $\Sigma_{g, 1}, \Sigma_{g^{\prime}, 1}$ as above. Then we get $\Sigma_{g, m+1}$, $\Sigma_{g^{\prime}, n+1}$. Note the boundaries of $\Sigma_{g, m+1}, \Sigma_{g^{\prime}, n+1}$ consist of $m+1$ circles, $n+1$ circles respectively. We decompose $\Sigma_{g, m+1}, \Sigma_{g^{\prime}, n+1}$ into

$$
(2 g+m-1) \text {-pair of pants } P_{i} \text { and } \quad\left(2 g^{\prime}+n-1\right) \text {-pair of pants } P_{i^{\prime}}^{\prime}
$$

such that $X_{i}=P_{i} \times S^{1}, Y_{i^{\prime}}=P_{i^{\prime}}^{\prime} \times S^{1}$. Recall that $X_{1}=P_{1} \times S^{1}$ meets $M_{2}$ and $Y_{1}=P_{1}^{\prime} \times S^{1}$ meets $M_{1}$.

As above we may have the generic fibration of $R\left(N_{\gamma}, S U(2)\right)^{-}$from the correspondence of $\Sigma_{g, m+1}$ and $\Sigma_{g^{\prime}, n+1}$ to $M_{1}$ and $M_{2}$. But the generic fibration of $R\left(N_{0},\left(n_{j}, n_{j^{\prime}}^{\prime}\right)\right)$ is not same as the generic fibration of $R\left(N_{\gamma},\left(n_{j}, n_{j^{\prime}}^{\prime}\right)\right)$ for $\gamma \neq 0$. If we consider boundaries of $P_{1}$ and $P_{1}^{\prime}$ around which the holonomies of $\left.\rho\right|_{P_{1}}$, $\left.\rho\right|_{P_{1}^{\prime}}$ are $\phi_{1}, \phi_{2}$ respectively, then we know that $\phi_{i}$ is fixed if $\gamma \neq 0$, is free with the above relation if $\gamma=0$. Hence we have generic fibrations of $R\left(N_{0},\left(n_{j}, n_{j^{\prime}}^{\prime}\right)\right)$, $R\left(N_{\gamma \neq 0},\left(n_{j}, n_{j^{\prime}}^{\prime}\right)\right)$ such that

$$
\begin{aligned}
& 0 \rightarrow \oplus_{i=1}^{l_{3}} T_{i}^{1} / \oplus_{i=1}^{l_{4}} Z_{2_{i}} \rightarrow R\left(N_{0}, S U(2),\left(n_{j}, n_{j^{\prime}}^{\prime}\right)\right) \rightarrow \oplus_{i=1}^{l_{5}(0)} \mathcal{L}_{i} \rightarrow 0 \\
& 0 \rightarrow \oplus_{i=1}^{l_{3}} T_{i}^{1} / \oplus_{i=1}^{l_{4}} Z_{2_{i}} \rightarrow R\left(N_{\gamma \neq 0}, S U(2),\left(n_{j}, n_{j^{\prime}}^{\prime}\right)\right) \rightarrow \oplus_{i=1}^{l_{5}(1)} \mathcal{L}_{i} \rightarrow 0
\end{aligned}
$$

where $l_{3}=3\left(g+g^{\prime}-1\right)+(m+n), l_{4}=2\left(g+g^{\prime}\right)+(m+n)-3$ and $l_{5}(0)=$ $3\left(g+g^{\prime}-1\right)+(m+n), l_{5}(1)=3\left(g+g^{\prime}\right)+(m+n)-4$.

To state our main result we define a function $\zeta_{A}^{k}(s)$ defined for $s \in C$ with $R e(s) \gg 0$, a finite set $A \in R-Z$ and for $k=0,1$. The function $\zeta_{A}^{k}(s)$ is defined by

$$
\zeta_{A}^{k}(s)=\sum_{n=1}^{\infty}\left(\frac{(-1)^{(n+1)^{k}}}{n^{s}} \prod_{i=1}^{m}\left|\frac{\sin \left(\pi n a_{i}\right)}{\sin \left(\pi a_{i}\right)}\right|\right)
$$

for $A=\left\{a_{1}, \cdots, a_{m}\right\}$. For an empty set $\phi, \zeta_{\phi}^{0}(s)$ is the Riemann-zeta function.

Theorem 4.3. The half density volume of $R\left(M,\left(n_{j}\right)\right)$ is given by

$$
\operatorname{Vol}\left(R\left(M,\left(n_{j}\right)\right)\right)=c_{M} \prod_{j=1}^{m} \frac{\left|\sin \left(\frac{\pi n_{j} \beta_{j}^{*}}{\alpha_{j}}\right)\right|}{\left|\alpha_{j}\right|^{\frac{1}{2}}} \zeta_{A_{M}}^{0}(2(g-1)+m)
$$

where

$$
\begin{aligned}
& c_{M}=2^{m-1} \operatorname{Vol}\left(S^{2}\right)^{m} \operatorname{Vol}(S U(2))^{-(g-1+m)} \\
& A_{M}=\left\{\frac{n_{1}}{\alpha_{1}}, \cdots, \frac{n_{m}}{\alpha_{m}}\right\} .
\end{aligned}
$$


The half density volume of $R\left(N_{\gamma},\left(n_{j}, n_{j^{\prime}}^{\prime}\right)\right)$ is given by

$$
\begin{aligned}
& \operatorname{Vol}\left(R\left(N_{0},\left(n_{j}, n_{j^{\prime}}^{\prime}\right)\right)\right) \\
& =c_{N}^{0} \prod_{j=1}^{m} \frac{\left|\sin \left(\frac{\pi n_{j} \beta_{j}^{*}}{\alpha_{j}}\right)\right|}{\left|\alpha_{j}\right|^{\frac{1}{2}}} \prod_{j^{\prime}=1}^{n} \frac{\left|\sin \left(\frac{\pi n_{j}^{\prime} \beta_{j^{\prime}}^{*}}{\alpha_{j^{\prime}}^{\prime}}\right)\right|}{\left|\alpha_{j^{\prime}}^{\prime}\right|^{\frac{1}{2}}} \zeta_{A_{N}}^{k}\left(2\left(g+g^{\prime}-1\right)+(m+n)\right)
\end{aligned}
$$

where

$$
\begin{aligned}
& c_{N}^{0}=2^{m+n+1} \operatorname{Vol}\left(S^{2}\right)^{m+n} \operatorname{Vol}(S U(2))^{-\left(g+g^{\prime}+m+n-1\right)}, \\
& k=\left\{\begin{array}{lll}
0 & \text { if } \quad \beta \psi_{2} \in Z \\
1 & \text { if } \quad \beta \psi_{2} \in Z\left[\frac{1}{2}\right]-Z,
\end{array}\right. \\
& A_{N}=\left\{2 \phi_{1}, 2 \phi_{2}, \frac{n_{1}}{\alpha_{1}}, \cdots, \frac{n_{m}}{\alpha_{m}}, \frac{n_{1}^{\prime}}{\alpha_{1}^{\prime}}, \cdots, \frac{n_{n}^{\prime}}{\alpha_{n}^{\prime}}\right\}
\end{aligned}
$$

and

$$
\begin{aligned}
& \operatorname{Vol}\left(R\left(N_{\gamma \neq 0},\left(n_{j}, n_{j^{\prime}}^{\prime}\right)\right)\right) \\
& =c_{N}^{1} \frac{1}{|\gamma|^{\frac{1}{2}}} \prod_{j=1}^{m} \frac{\left|\sin \left(\frac{\pi n_{j} \beta_{j}^{*}}{\alpha_{j}}\right)\right|}{\left|\alpha_{j}\right|^{\frac{1}{2}}} \prod_{j^{\prime}=1}^{n} \frac{\left|\sin \left(\frac{\pi n_{j}^{\prime} \beta_{j^{\prime}}^{*}}{\alpha_{j^{\prime}}^{\prime}}\right)\right|}{\left|\alpha_{j^{\prime}}^{\prime}\right|^{\frac{1}{2}}} \zeta_{A_{M_{1}}}^{0}(2 g+m-1) \zeta_{A_{M_{2}}}^{0}\left(2 g^{\prime}+n-1\right)
\end{aligned}
$$

where

$$
\begin{aligned}
& c_{N}^{1}=2^{m+n+1} \operatorname{Vol}\left(S^{2}\right)^{m+n+2} \operatorname{Vol}(S U(2))^{-\left(g+g^{\prime}+m+n\right)}, \\
& A_{M_{1}}=\left\{2 \phi_{1}, \frac{n_{1}}{\alpha_{1}}, \cdots, \frac{n_{m}}{\alpha_{m}}\right\}, \quad A_{M_{2}}=\left\{2 \phi_{2}, \frac{n_{1}^{\prime}}{\alpha_{1}^{\prime}}, \cdots, \frac{n_{n}^{\prime}}{\alpha_{n}^{\prime}}\right\} .
\end{aligned}
$$

Proof. We shall compute only the half density volume of $R\left(N_{\gamma},\left(n_{j}, n_{j^{\prime}}^{\prime}\right)\right)$. For $R\left(M,\left(n_{j}\right)\right)$ we can get the result by the same way.

From the proposition 3.3 and (4.2) we have that

$$
\begin{aligned}
& \operatorname{Vol}\left(R\left(N_{\gamma},\left(n_{j}, n_{j^{\prime}}^{\prime}\right)\right)\right)=\int_{R\left(N_{\gamma},\left(n_{j}, n_{j^{\prime}}^{\prime}\right)\right)} \tau\left(N_{\gamma}, \operatorname{Ad}\left(\rho_{N_{\gamma}}\right)\right)^{\frac{1}{2}} \\
& =2^{m+n} f(\gamma)^{\frac{1}{2}} \prod_{j=1}^{m} \frac{\left|\sin \left(\frac{\pi n_{j} \beta_{j}^{*}}{\alpha_{j}}\right)\right|}{\left|\alpha_{j}\right|^{\frac{1}{2}}} \prod_{j^{\prime}=1}^{n} \frac{\left|\sin \left(\frac{\pi n_{j^{\prime}}^{\prime} \beta_{j}^{\prime *}}{\alpha_{j^{\prime}}^{\prime}}\right)\right|}{\left|\alpha_{j^{\prime}}^{\prime}\right|^{\frac{1}{2}}} \int_{R\left(N_{\gamma},\left(n_{j}, n_{j^{\prime}}^{\prime}\right)\right)} D_{H_{1}\left(N_{\gamma}, s u(2)_{\rho_{N}}\right)} \\
& =2^{m+n} f(\gamma)^{\frac{1}{2}} \prod_{j=1}^{m} \frac{\left|\sin \left(\frac{\pi n_{j} \beta_{j}^{*}}{\alpha_{j}}\right)\right|}{\left|\alpha_{j}\right|^{\frac{1}{2}}} \prod_{j^{\prime}=1}^{n} \frac{\left|\sin \left(\frac{\pi n_{j^{\prime}}^{\prime} \beta_{j}^{\prime *}}{\alpha_{j^{\prime}}^{\prime}}\right)\right|}{\left|\alpha_{j^{\prime}}^{\prime}\right|^{\frac{1}{2}}} \int_{F} \mu_{F} \cdot \int_{B_{\gamma}} \mu_{B_{\gamma}}
\end{aligned}
$$


where $F=\oplus_{i=1}^{l_{3}} T_{i}^{1} / \oplus_{i=1}^{l_{4}} Z_{2_{i}}, B_{\gamma}=\oplus_{i=1}^{l_{5}(k)} \mathcal{L}_{i}$ with $k=0,1$ if $\gamma=0, \gamma \neq 0$ and $\mu_{F}, \mu_{B_{\gamma}}$ are the volume forms of $F, B_{\gamma}$ such that $D_{H_{1}\left(N_{\gamma}, s u(2)_{\rho_{N}}\right)}=\mu_{F} \cdot p^{*} \mu_{B_{\gamma}}$. We see easily that $\mu_{F}$ on $F$ is the $3\left(g+g^{\prime}-1\right)+(m+n)$ copies of the natural volume form $\nu$ of the maximal torus $T^{1}$ with $\int_{T^{1}} \nu=1$. In fact $\mu_{F}$ is given by the volume form of the kernel of $\delta_{0}$ in the proof of the proposition 3.3. So we have

$$
\int_{F} \mu_{F}=\frac{1}{2^{2\left(g+g^{\prime}\right)+(m+n)-3}} \text {. }
$$

The volume form $\mu_{B_{\gamma}}$ on $B_{\gamma}=\oplus_{i=1}^{l_{5}(k)} \mathcal{L}_{i}$ is given by the volume form of the cokernel of $\delta_{1}$ in the proof of the proposition 3.3. So $\mu_{B_{\gamma}}$ can be written by $f_{0} \nu_{1} \cdots \nu_{l_{5}(0)}$ or $f_{1} \nu_{0} \cdots \nu_{l_{5}(1)}$ for some $f_{k} \in L^{2}\left(\oplus_{i=1}^{l_{5}(k)} \mathcal{L}_{i}\right)$ for $k=0,1$. ¿From (2.4), the proposition 2.5 and the pants decomposition of $\Sigma_{g, m+1}, \Sigma_{g^{\prime}, n+1}$, we can see that $f_{0}=f \cdot g, f_{1}=f \cdot h$ where $f$ is the product of

$$
\begin{aligned}
& 2\left(g+g^{\prime}-2\right)-\text { copies of } \frac{2}{\operatorname{Vol}(S U(2))^{2}} \sum_{\alpha} \frac{1}{n_{\alpha}} \prod_{i=1}^{3} \chi_{\alpha}(\cdot), \\
& m-\text { copies of } \frac{2 \operatorname{Vol}\left(S^{2}\right)}{\operatorname{Vol}(S U(2))^{2}} \sum_{\alpha} \frac{1}{n_{\alpha}} \frac{\left|\sin \left(\frac{\pi n_{\alpha} n_{j}}{\alpha_{j}}\right)\right|}{\left|\sin \left(\frac{\pi n_{j}}{\alpha_{j}}\right)\right|} \chi_{\alpha}(\cdot) \chi_{\alpha}(\cdot), \\
& n-\text { copies of } \frac{2 \operatorname{Vol}\left(S^{2}\right)}{\operatorname{Vol}(S U(2))^{2}} \sum_{\alpha} \frac{1}{n_{\alpha}} \frac{\left|\sin \left(\frac{\pi n_{\alpha} n_{j^{\prime}}^{\prime}}{\alpha_{j^{\prime}}^{\prime}}\right)\right|}{\left|\sin \left(\frac{\pi n_{j^{\prime}}^{\prime}}{\alpha_{j^{\prime}}^{\prime}}\right)\right|} \chi_{\alpha}(\cdot) \chi_{\alpha}(\cdot),
\end{aligned}
$$

and $g$ is the product of

$$
2 \text { - copies of } \frac{2}{\operatorname{Vol}(S U(2))^{2}} \sum_{\alpha} \frac{1}{n_{\alpha}} \prod_{i=1}^{3} \chi_{\alpha}(\cdot),
$$

and $h$ is the product of

$$
\begin{aligned}
& \frac{2 \operatorname{Vol}\left(S^{2}\right)}{\operatorname{Vol}(S U(2))^{2}} \sum_{\alpha} \frac{1}{n_{\alpha}} \frac{\left|\sin \left(2 \pi n_{\alpha} \phi_{1}\right)\right|}{\left|\sin \left(2 \pi \phi_{1}\right)\right|} \chi_{\alpha}(\cdot) \chi_{\alpha}(\cdot), \\
& \frac{2 \operatorname{Vol}\left(S^{2}\right)}{\operatorname{Vol}(S U(2))^{2}} \sum_{\alpha} \frac{1}{n_{\alpha}} \frac{\left|\sin \left(2 \pi n_{\alpha} \phi_{2}\right)\right|}{\left|\sin \left(2 \pi \phi_{2}\right)\right|} \chi_{\alpha}(\cdot) \chi_{\alpha}(\cdot) .
\end{aligned}
$$

where the value of representation $\rho_{N_{\gamma}}\left(\left[x_{i}^{\cdot}\right]\right)$ or $\rho_{N_{\gamma}}\left(\left[y_{i^{\prime}}\right]\right)$ appears at the slot of $\chi_{\alpha}(\cdot)$ if $x_{i}$ or $y_{i^{\prime}}$ is a homotopy class of a boundary of $P_{i}$ or $P_{i^{\prime}}^{\prime}$. 
We use the orthogonal pairing of the characters on each intersection circle between a pair of pants such that

$$
\int_{\mathcal{L}_{i}} \chi_{\alpha}\left(\rho_{N}(\cdot)\right) \chi_{\beta}\left(\rho_{N}(\cdot)^{-1}\right) \nu_{i}=\delta_{\alpha, \beta} \operatorname{Vol}(S U(2))
$$

These pairings on $\mathcal{L}_{i}$ for $1 \leq i \leq l_{5}(1)$ with respect to $\nu_{i}$ give $\int_{B_{\gamma}} \mu_{B_{\gamma}}$ for $\gamma \neq 0$ so that we get the volume of $R\left(N_{\gamma},\left(n_{j}, n_{j^{\prime}}^{\prime}\right)\right)$ when $\gamma \neq 0$. Note that this pairing does not occur on the intersection circle between $P_{1}$ and $P_{1}^{\prime}$ since the holonomies $\phi_{1}$ and $\phi_{2}$ are fixed if $\gamma \neq 0$.

When $\gamma=0$ the above pairings on $\mathcal{L}_{i}$ with respect to $\nu_{i}$ give same result except one $\mathcal{L}_{i}$ which comes from the intersection of $P_{1}$ and $P_{1}^{\prime}$. This is because the holonomies of $\rho_{N_{\gamma}}$ around a boundary of $P_{1}$ and $P_{1}^{\prime}$ which give the pairing do not coincide but have the relation $\phi_{1}= \pm \phi_{2}+\beta \psi_{2}$. So the pairing in this case is given by

$$
\int_{\mathcal{L}_{i}} \chi_{\alpha}\left(\rho_{N}\left(D\left(e^{2 \pi \phi_{1}}\right)\right)\right) \chi_{\beta}\left(\rho_{N}\left(D\left(e^{2 \pi \phi_{2}}\right)\right)^{-1}\right) \nu_{i}=(-1)^{k} \delta_{\alpha, \beta} \operatorname{Vol}(S U(2))
$$

where $D\left(e^{2 \pi \phi_{i}}\right)$ is the $S U(2)$-matrix with the diagonal elements $\left\{e^{2 \pi \phi_{i}}, e^{-2 \pi \phi_{i}}\right\}$ and

$$
k= \begin{cases}0 & \text { if } \quad \beta \psi_{2} \in Z \\ 1 & \text { if } \quad \beta \psi_{2} \in Z\left[\frac{1}{2}\right]-Z .\end{cases}
$$

Recall that $\beta$ is given in (3.1). We have that $\left\{n_{\alpha}\right\}$ is the set of the natural numbers for the Lie group $S U(2)$. For this see the proposition (5.3) of [B,tD]. The constants $C_{N}^{0}, C_{N}^{1}$ are given by gathering all the constants in the above pairings.

We remark some facts about theorem 4.3. We compute the half density volumes of $R\left(M,\left(n_{j}\right)\right), R\left(N_{\gamma},\left(n_{j}, n_{j^{\prime}}^{\prime}\right)\right)$ using the fibration structure of the representation space $R\left(\Sigma_{g, m},\left(n_{j}\right)\right)$ which has the symplectic structure. In [W2] Witten computes the symplectic volume of $R\left(\Sigma_{g, m},\left(n_{j}\right)\right)$. We use his method with some modification. So we point out two differences between the symplectic volume form - this is the R-torsion of $\Sigma_{g, m}$ - of $R\left(\Sigma_{g, m},\left(n_{j}\right)\right)$ and the half density of $R\left(M,\left(n_{j}\right)\right)$. The first comes from two different volume forms of the fiber $\oplus_{i=1}^{3(g-1)+m} T_{i}^{1} / \oplus_{i=1}^{2 g+m-3} Z_{2_{i}}$. In our case the volume form over the fiber comes from the natural volume form $\nu$ on the maximal torus $T^{1}$ of $S U(2)$, but in [W2] the fiber volume form comes from the volume form $\nu_{0}$ induced from the volume form of $S U(2)$. Of course the difference comes from the different constructions of two volume forms. The second 
is the term $\sin \left(\pi \frac{n_{j}}{\alpha_{j}}\right)$. In [W2] this term is necessary to define natural symplectic volume form of $R\left(\Sigma_{g, m},\left(n_{j}\right)\right)$ so that this term cancels out a factor of character term $\frac{\sin \left(\frac{\pi n n_{j}}{\alpha_{j}}\right)}{\sin \left(\frac{\pi n_{j}}{\alpha_{j}}\right)}$ in [W2]. But we need not this term to give the cancellation.

\section{$\S 5$ Application to Jeffrey-Weitsman-Witten invariant}

In this section we apply our result of the previous section to compute the JeffreyWeitsman-Witten invariant of a Seifert fibred manifold $M$ with base surface $\Sigma_{g \geqq 2}$. In this case the irreducible $S U(2)$-representation space $R(M, S U(2))^{-}$is nondiscrete set so that the R-torsion is used as the half density of $R(M, S U(2))^{-}$ in defining the Jeffrey-Weitsman-Witten invariant.

We review the Chern-Simons gauge theory to understand the definition of the Jeffrey-Weitsman-Witten invariant. For the detail of the Chern-Simons gauge theory, see $[\mathrm{R}, \mathrm{S}, \mathrm{W}],[\mathrm{J}, \mathrm{W} 1]$.

Let $X$ be a 2-dimensional manifold and $P$ be a principal $S U(2)$-bundle over $X$. Let $\mathcal{A}, \mathcal{A}_{F}, \mathcal{G}$ be the affine space of connection one forms of $P$, the space of flat connections of $P$ and the gauge transformation group of $P$ respectively. Let $\mathcal{M}_{2}$ be the moduli space of the flat connections of $P$.

We consider a 3-dimensional manifold $Y_{1}$ with a boundary $X$. Moreover we assume that a neighborhood of $X$ in $Y_{1}$ is diffeomorphic to $X \times[0,1)$. For $A \in$ $\mathcal{A}, g \in \mathcal{G}$ we consider a $U(1)$-valued function $\mathcal{S}(A, g)$ defined by

$$
\mathcal{S}(A, g) \equiv \exp \left(2 \pi i\left(C S\left(\tilde{A}^{\tilde{g}}\right)-C S(\tilde{A})\right)\right)
$$

where $\tilde{A}$ and $\tilde{g}$ are the extensions of $A$ and $g$ into $Y_{1}, \tilde{A}^{\tilde{g}}$ is the gauge transformation of $\tilde{A}$ by $\tilde{g}$ and the Chern-Simons invariant $C S(\tilde{A})$ is given by

$$
C S(\tilde{A})=\frac{1}{8 \pi^{2}} \int_{Y_{1}} \operatorname{tr}\left(d \tilde{A} \wedge \tilde{A}+\frac{2}{3} \tilde{A} \wedge \tilde{A} \wedge \tilde{A}\right) .
$$

Such an extension of $\tilde{g}$ always exists since $\pi_{1}(S U(2))=\pi_{2}(S U(2))=0$. We choose the extensions so that $\tilde{A}$ and $\tilde{g}$ are pull-backs of $A$ and $g$ by the projection to $X$ over $X \times[0,1)$ respectively. Then $\mathcal{S}$ is independent on the extension $Y_{1}$ and the extension $\tilde{A}$ and $\tilde{g}$. In fact the extensions $\left(\tilde{A}_{1}, \tilde{g}_{1}\right)$ and $\left(\tilde{A}_{2}, \tilde{g}_{2}\right)$ into $Y_{1}$ and $Y_{2}$ give a connection $\tilde{B}$ and a gauge transformation $\tilde{h}$ on $Y=Y_{1} \cup Y_{2}$ so that

$$
\begin{aligned}
& \exp \left(2 \pi i\left(C S\left(\tilde{A}_{1}^{\tilde{g}}\right)-C S\left(\tilde{A}_{1}\right)\right)\right) \exp \left(2 \pi i\left(C S\left(\tilde{A}_{2}^{\tilde{g}}\right)-C S\left(\tilde{A}_{2}\right)\right)\right)^{-1} \\
& =\exp \left(2 \pi i\left(C S\left(\tilde{B}^{\tilde{h}}\right)-C S(\tilde{B})\right)\right)=1 .
\end{aligned}
$$


The above function $\mathcal{S}$ over $\mathcal{A} \times \mathcal{G}$ is a cocycle since

$$
\mathcal{S}(\tilde{A}, \tilde{g}) \mathcal{S}\left(\tilde{A}^{\tilde{g}}, \tilde{h}\right)=\mathcal{S}(\tilde{A}, \tilde{g} \tilde{h}) .
$$

We can define a line bundle $\mathcal{L}$ over $\mathcal{M}_{2}$ by

$$
\mathcal{L} \equiv \mathcal{A}_{F} \times{ }_{\mathcal{S}} C
$$

where the right side is the quotient space given by the equivalence relation

$$
(A, z) \sim\left(A^{g}, \mathcal{S}(A, g) z\right)
$$

for $A \in \mathcal{A}_{F}, z \in C$.

We consider a 3-dimensional manifold $Y$ and a principal $S U(2)$-bundle $P_{Y}$ over $Y$. We decompose $Y$ into two handle bodies $Y_{1}$ and $Y_{2}$. Let $X$ be the intersection of $Y_{1}$ and $Y_{2}$. We apply above construction to 2-dimensional manifold $X$ and $\left.P_{Y}\right|_{X}=P$. We consider the restriction of line bundle $\mathcal{L}$ to Lagrangian submanifolds $L_{1}, L_{2}$ of $\mathcal{M}_{2}$ where $L_{1}, L_{2}$ are made from the handle bodies $Y_{1}, Y_{2}$ of $Y$. Let $\mathcal{L}_{i}$ be the restriction of the line bundle $\mathcal{L}$ to $L_{i}$. Then there is section $\mathcal{S}_{i}(A)$ of $\mathcal{L}_{i}$ over $L_{i}$ defined by

$$
\mathcal{S}_{i}(A)=\exp \left(2 \pi i C S\left(A_{Y_{i}}\right)\right)
$$

for $[A] \in \mathcal{M}_{2}$ and $A_{Y_{i}}$ is an extension of $A$ to $Y_{i}$.

Now we consider the intersection of the two Lagrangian submanifolds $L_{1}, L_{2}$ in $\mathcal{M}_{2}$. Then we can see that this intersection is the moduli space of flat connections of $P_{Y}$ over $Y$ which we denote by $\mathcal{M}_{3}$. By the correspondence between the flat connection $A$ and the $S U(2)$-representation $\rho_{Y}$ of $\pi_{1}(Y), \mathcal{M}_{3}$ can be identified with $R(Y, S U(2))$. There may occur singularities of $\mathcal{M}_{2}$ within $\mathcal{M}_{3}$. But the set of singularities is a measure zero set in $\mathcal{M}_{3}$. So we may not care these singularities in the following construction since we shall integrate over the dense subset $R(Y, S U(2))^{-}$of $\mathcal{M}_{3}=R(Y, S U(2))$.

We consider the k-tensor power of $\mathcal{L}, \mathcal{L}^{\otimes^{k}}$ over $\mathcal{M}_{2}$ and their restrictions to two Lagrangian submanifolds $L_{1}, L_{2}$. We denote these by $\mathcal{L}_{1}^{\otimes^{k}}, \mathcal{L}_{2}^{\otimes^{k}}$. Then we can pair two sections $\mathcal{S}_{i}^{k}=\mathcal{S}_{i}^{\otimes^{k}}$ of $\mathcal{L}_{i}^{\otimes^{k}}$ in $\mathcal{M}_{3}$ by the hermitian product of the complex line $C$. We denote this pairing by $\left\langle\mathcal{S}_{1}^{k}, \mathcal{S}_{2}^{k}\right\rangle$. Then this can be considered as an $U(1)$ valued function on $\mathcal{M}_{3}=R(Y, S U(2))$. We can see easily that $\left\langle\mathcal{S}_{1}^{k}, \mathcal{S}_{2}^{k}\right\rangle$ at a connection $A$ is the exponential of the Chern-Simons invariant of $Y$, that is, $\exp (2 k \pi i C S(A))$.

We recall that the half density derived from the R-torsion- $\tau\left(Y, A d\left(\rho_{Y}\right)\right)^{\frac{1}{2}}$ can be considered as a measure of $R(Y, S U(2))^{-}$. The Jeffrey-Weitsman-Witten invariant is defined by integrating the pairing $\left\langle\mathcal{S}_{1}^{k}, \mathcal{S}_{2}^{k}\right\rangle$ with respect to half density $\tau\left(Y, A d\left(\rho_{Y}\right)\right)^{\frac{1}{2}}$ over $R(Y, S U(2))^{-}$using the correspondence between the flat connection $A$ of $P$ and the $S U(2)$-representation $\rho_{Y}$ of $\pi_{1}(Y)$. We formulate this construction as the following definition. 
Definition 5.1. For integer $k$, the Jeffrey-Weitsman-Witten invariant $Z(Y, k)$ is defined by

$$
Z(Y, k)=\int_{R(Y, S U(2))^{-}}\left\langle\mathcal{S}_{1}^{k}, \mathcal{S}_{2}^{k}\right\rangle \tau\left(Y, A d\left(\rho_{Y}\right)\right)^{\frac{1}{2}} .
$$

This definition is given in [J,W1]. This definition is motivated from the asymptotic expansion of the Witten invariant $Z_{Y}(k)$ of 3-dimensional manifold $Y$ [W1]. The asymptotic expansion of $Z_{Y}(k)$ is given by

$$
Z_{Y}(k) \simeq \frac{1}{2} \sum_{i}\left(\tau\left(Y, A_{i}\right)\right)^{\frac{1}{2}} \exp \left(-\frac{3 \pi i+2 \pi i S F\left(A_{i}\right)}{4}\right) \exp \left(2(k+2) \pi i C S\left(A_{i}\right)\right)
$$

where the sum is taken over the finite set of flat connections $A_{i}, \tau\left(Y, A_{i}\right)$ is the Reidemeister torsion for $A_{i}$ of $Y$ and $S F\left(A_{i}\right)$ is the spectral flow from trivial connection to the flat connection $A_{i}$. The above formula is given in $[\mathrm{F}, \mathrm{G}]$. We can see that if the moduli space of flat connections is a discrete set, then definition 5.1 is almost same as the leading term of the above asymptotic expansion since the square root of the R-torsion becomes a point mass in this case.

Now we compute the Jeffrey-Weitsman-Witten invariant $Z(M, k)$ of Seifert fibred manifold $M$ with the non-discrete irreducible $S U(2)$-representation space $R(M, S U(2))^{-}$by applying the previous result. To compute $Z(Y, k)$, we must integrate the pairing $<\mathcal{S}_{1}^{k}, \mathcal{S}_{2}^{k}>$ with respect to the half density of the R-torsion over $R(M, S U(2))^{-}$. We know that the value $<\mathcal{S}_{1}^{k}, \mathcal{S}_{2}^{k}>$ at the flat connection $A$ is simply the Chern-Simons invariant of $A$. This invariant is constant in each connected component $R\left(M,\left(n_{j}\right)\right)$. By the result of $[\mathrm{A}]$, the value for the fixed connected component $R\left(M,\left(n_{j}\right)\right)$ is given by

$$
\exp \left[2 k \pi i\left(-\sum_{j=1}^{m}\left(\frac{\beta_{j}^{*} n_{j}^{2}}{\alpha_{j}}+\frac{2 \epsilon n_{j}}{\alpha_{j}}\right)+\epsilon^{2} \sum_{j=1}^{m} \frac{\beta_{j}}{\alpha_{j}}\right)\right]
$$

where $\epsilon=\frac{1}{2}, 1$ if $\rho_{M}(h)=-1,1$ and $\beta_{j} \beta_{j}^{*}=1(\bmod ) \alpha_{j}$ as above.

So the value of the Jeffrey-Weitsman-Witten invariant $Z(Y, k)$ over the connected component $R\left(M,\left(n_{i}\right)\right)$ is given by

$$
\begin{aligned}
& \int_{R\left(M,\left(n_{j}\right)\right)}<\mathcal{S}_{1}^{k}, \mathcal{S}_{2}^{k}>\left(\tau\left(M, A d\left(\rho_{M}\right)\right)\right)^{\frac{1}{2}} \\
& =2^{m}<\mathcal{S}_{1}^{k}, \mathcal{S}_{2}^{k}>\prod_{j=1}^{m} \frac{\left|\sin \left(\frac{\pi n_{j} \beta_{j}^{*}}{\alpha_{j}}\right)\right|}{\left|\alpha_{j}\right|^{\frac{1}{2}}} \int_{R\left(M,\left(n_{j}\right)\right)} D_{H_{1}\left(M, s u(2)_{\rho_{M}}\right)}
\end{aligned}
$$

So we have the following theorem from the theorem 4.3 and (5.2). 
Theorem 5.3. For the integer $k$, the Jeffrey-Weitsman-Witten invariant $Z(M, k)$ of the Seifert fibred manifold $M\left(g,\left(\alpha_{1}, \beta_{1}, \ldots, \alpha_{m}, \beta_{m}\right)\right)$ is given by

$$
\begin{aligned}
c_{M} \sum_{\left\{\left(n_{j}\right)\right\}} & \exp \left[2 k \pi i\left(-\sum_{j=1}^{m}\left(\frac{\beta_{j}^{*} n_{j}^{2}}{\alpha_{j}}+\frac{2 \epsilon n_{j}}{\alpha_{j}}\right)+\epsilon^{2} \sum_{j=1}^{m} \frac{\beta_{j}}{\alpha_{j}}\right)\right] \\
& \times \prod_{j=1}^{m} \frac{\left|\sin \left(\frac{\pi n_{j} \beta_{j}^{*}}{\alpha_{j}}\right)\right|}{\left|\alpha_{j}\right|^{\frac{1}{2}}} \sum_{n=1}^{\infty}\left(\frac{1}{n^{2(g-1)+m}} \prod_{j=1}^{m} \frac{\left|\sin \left(\frac{\pi n n_{j}}{\alpha_{j}}\right)\right|}{\left|\sin \left(\frac{\pi n_{j}}{\alpha_{j}}\right)\right|}\right)
\end{aligned}
$$

where the above sum is taken over the finite set of the rotation numbers $\left\{\left(n_{j}\right)\right\}$, $c_{M}=2^{m-1} \operatorname{Vol}\left(S^{2}\right)^{m} \operatorname{Vol}(S U(2))^{g+m-1}$ and $\epsilon=\frac{1}{2}$ or 1 if $\rho_{M}(h)=-1$ or 1.

We can see that $Z(M, k)$ depends only on the manifold $M$ via the Seifert invariant $\left(g ;\left(\alpha_{i}, \beta_{i}\right)\right)$ since the set of all the rotation numbers $\left(n_{j}\right)$ is determined by $\pi_{1}(M)$.

\section{REFERENCES}

[A] D.R.Auckly, Topological methods to compute Chern-Simons invariants, Math. Proc. Camb. Phil. Soc. 115 (1994), 229-251.

[B,tD] T.Bröcker and T.tom Dieck, Representations of Compact Lie Groups, Springer-Verlag, New York Inc., 1985.

[F] D.S.Freed, Reidemeister torsion, spectral sequences, and Brieskorn spheres, J.reine angew.Math. 429 (1992), 75-89.

[F,G] D.S.Freed and R.E.Gompf, Computer calculation of Witten's 3-manifold invariant, Commun.Math.Phys. 141 (1991), 79-117.

[F,S] R.Fintushel and R.J.Stern, Instanton Homology of Seifert Homology three spheres, Proc. London. Math. Soc. (3) 61 (1990), 109-137.

[J,W1] L.C.Jeffrey and J.Weitsman, Half density Quantization of the moduli space of flat connections and Witten's semiclassical manifold invariants, Topology 32 (1993), 509-529.

[J,W2] L.C.Jeffrey and J.Weitsman, Toric Structures on the Moduli Space of Flat Connections on a Riemann Surface:Volumes and the Moment Map, Advances In Mathematics 106 (1994), 151-168.

[K,K] P.Kirk and E.Klassen, Chern-Simons Invariant of 3-Manifolds decomposed along Tori and the Circle Bundle over the Representation Space of $T^{2}$, Commun.Math.Phys. 153 (1993), 521-557.

[R,S,W] T.R.Ramadas, I.M.Singer and J.Weitsman, Some Comments on Chern-Simons Gauge theory, Commun.Math.Phys. 126 (1989), 409-420.

[W1] E.Witten, Quantum Field Theory and the Jones Polynomial, Commun.Math.Phys. 121 (1989), 351-399.

[W2] E.Witten, On Quantum Gauge Theories in Two Dimensions, Commun.Math.Phys. 141 (1991), 153-209.

e-mail address:jinpark@alliant.snu.ac.kr 